\title{
Data Mining as Constraint Logic Programming ${ }^{\star}$
}

\author{
Luc De Raedt \\ Institut für Informatik, Albert-Ludwig-University \\ Georghes Koehler Allee 79, D-79110 Freiburg, Germany \\ Email: deraedt@informatik.uni-freiburg.de
}

\begin{abstract}
An inductive database allows one to query not only the data but also the patterns of interest. A novel framework, called RDM, for inductive databases is presented. It is grounded in constraint logic programming. RDM provides a small but powerful set of of built-in constraints to query patterns. It is also embedded in the programming language Prolog. In this paper, the semantics of RDM is defined and a solver is presented. The resulting query language allows us to declaratively specify the patterns of interest, the solver then takes care of the procedural aspects.
\end{abstract}

\section{Introduction}

Imielinski and Mannila [17] present a database perspective on knowledge discovery. From this perspective, data mining is regarded as a querying process to a database mining system. Querying for knowledge discovery requires an extended query language (w.r.t. database languages), which supports primitives for the manipulation, mining and discovery of rules, as well as data. The integration of such rule querying facilities provides new challenges for database technology.

The view of Imielinski and Mannila is very much in the spirit of Kowalski's celebrated equation "Algorithm = Logic + Control" [19]. Indeed, inductive database queries declaratively specify the logic of the problem and the inductive database management system should provide the procedures (i.e. the control) for solving the query. Thus the hope is that inductive databases will allow us to nicely separate the declarative from the procedural aspects.

Mannila and Toivonen [23] formulate the general pattern discovery task as follows. Given a database $r$, a language $\mathcal{L}$ for expressing patterns, and a constraint $q$, find the theory of $r$ with respect to $\mathcal{L}$ and $q$, i.e. $\operatorname{Th}(\mathcal{L}, r, q)=\{\phi \in$ $\mathcal{L} \mid q(r, \phi)$ is true $\}$. This formulation of pattern discovery is generic in that it makes abstraction of several specific tasks including the discovery of association rules, frequent patterns, inclusion dependencies, functional dependencies, frequent episodes, ... Also, efficient algorithms for solving these tasks are known [23].

So far, the type of constraint that has been considered is rather simple and typically relies on the frequency of patterns. However, knowledge discovery is often regarded as a cyclic and iterative process [12], where one considers a

\footnotetext{
* This paper significantly extends $[8,9]$.
} 
number of different selection predicates, patterns, example sets and versions of the database. Therefore, there is a need for considering and combining different theories $T h\left(\mathcal{L}_{i}, q_{i}, r_{i}\right)$. The practical necessity to combine various theories $\operatorname{Th}\left(\mathcal{L}_{i}, q_{i}, r_{i}\right)$ forms one of the main motivations underlying the work on inductive databases [24] and database mining query languages [17, 18, 26, 14, 13]. Indeed, a number of extensions to database languages such as SQL have been introduced with the aim of supporting the generation and manipulation of theories $\operatorname{Th}\left(\mathcal{L}_{i}, q_{i}, r_{i}\right)$.

The need to combine various constraints and answers also arises in constraint programming [15]. We pursue this analogy further by developing a constraint logic programming language RDM to support data mining. RDM stands for Relational Database Mining. There are different versions of RDM, which depend on the pattern domain under consideration. The pattern domains considered here include item-sets [1], sequences [20, 21], graphs [30] and Datalog queries [7]. Nevertheless, the key primitives and the execution mechanism of RDM are presented at a domain independent level. RDM supports a larger variety of constraints than previous inductive databases. In addition to providing an operator to manipulate patterns, it also allows the user to manipulate sets of examples, and provides selection predicates that impose a minimum or maximum frequency threshold. This allows RDM to address descriptive as well as predictive induction. In descriptive induction one is interested in frequent patterns, whereas in predictive induction, one aims at discovering patterns that are frequent on the positive examples but infrequent on the negative ones. Further primitives in $\mathrm{RDM}$ are based on the notion of generality (among patterns) and on coverage (w.r.t. specific examples). Queries in RDM consist of a number of primitives which specify constraints on the patterns of interest. Each constraint results in a theory $\operatorname{Th}\left(\mathcal{L}_{i}, q_{i}, r_{i}\right)$. RDM then efficiently computes the intersection of these theories. RDM's execution mechanism is based on the level-wise version space algorithm of [10], which integrates the version space approach by Tom Mitchell [28] with the level-wise algorithm [23]. Directly computing the intersection of the theories contrasts with the approach taken in the MINE RULE operator by Meo et al. [26] and the approach of Boulicaut et al. [3] in that these latter approaches typically generate one theory $T h\left(\mathcal{L}_{i}, q_{i}, r_{i}\right)$ and then repeatedly modify it.

Embedding RDM within a programming language such as Prolog [4] puts database mining on the same methodological grounds as constraint programming. Indeed, each of the different selection predicates or primitives in a query or program imposes certain constraints on the patterns. As in constraint programming, we have to specify the semantics of these primitives as well as develop efficient solvers for queries and programs. In this paper, an operational semantics for RDM is specified and an efficient solver presented.

The paper is organised as follows: in Section 2, we define the basic RDM primitives, in Section 3, we show RDM at work through a number of database mining queries, in Section 4, we present a solver for simple queries, in Section 5, we discuss how to extend the language and the solver, and finally, in Section 6, we conclude and touch upon related work. 


\section{Data mining domains}

\subsection{Data mining domains}

In data mining various pattern domains are employed. A pattern domain consists of three components: a set of patterns, a set of observations or examples and a set of constraints. The most fundamental constraint is the coverage constraint, which specifies when a pattern matches (covers) an example.

For illustration, consider the following pattern domains:

Definition 1. (Item-Sets IS [1]) Consider the a set of all possible items $\mathcal{I}$. An item-set $i$ is then a subset of $\mathcal{I}$. Examples (sometimes called transactions) and patterns are item-sets. A pattern $p$ covers an example $e$ if and only if $p \subseteq e$.

Items are elements of interest. Typical examples of items are products. The set $\mathcal{I}$ then corresponds to the set of all products in a given supermarket and an item-set to a possible transaction.

Definition 2. (Sequences SEQ) Examples and patterns are sequences. A pattern covers an example if it is a subsequence of the example.

Definition 3. (Graphs G [30]) Examples as well as patterns are labeled graphs. A pattern $p$ covers an example $e$ if and only if $p$ is isomorphic to a subgraph of $e$.

Definition 4. (Datalog Queries DQ [7]) A database $\mathcal{D}$ is given. The database contains the predicate $k e y / 1$. An example is then a substitution $\{K \leftarrow k\}$ such that $k$ is a constant and $\mathcal{D}=k e y(k)$. A pattern is a Datalog query of the form $?-\operatorname{key}(K), l_{1}, \ldots, l_{n}$, where the $l_{i}$ are literals. A pattern $p$ covers an example $\theta$ in $\mathcal{D}$ if $\mathcal{D}=p \theta$.

Throughout the paper we will employ the domain of item-sets to illustrate our inductive database formalism RDM. However, we wish to stress that all of the concepts introduced equally apply to the other domains (SEQ, G, and DQ).

\subsection{Data types}

The RDM framework distinguishes three basic data types:

- $\mathcal{E}$ : denotes the example data type; depending on the application domain this can be item-sets, graphs, sequences, keys, ...

- $\mathcal{P}$ : denotes the pattern data type; depending on the application domain this can be item-sets, graphs, sequences, Datalog queries, ...

- $\mathcal{D}(\mathcal{T})$ : denotes the data set type over the type $\mathcal{T}$; datasets of type $\mathcal{D}(\mathcal{E})$ are sets of examples of type $\mathcal{E}$; datasets of type $\mathcal{D}(\mathcal{P})$ are sets of patterns of type $\mathcal{P}$. 
In many situations the data types $\mathcal{E}$ and $\mathcal{P}$ will be identical. Throughout the rest of the paper we will make abstraction of how specific data types are internally represented. This will leave a maximum degree of freedom to the implementer. We do however assume that the membership predicate is available:

- ?Element in + Set : succeeds when Element $\in$ Set.

We employ the usual conventions where we list the modes of the predicates using " - "," + "and "?": " - arguments must not be instantiated," + " arguments must be instantiated to a non-variable term, and "?" arguments may but need not be instantiated at the time of calling.

Let us illustrate the types on the domain of item-sets IS. $\mathcal{E}$ as well as $\mathcal{P}$ could be represented as ordered lists of atoms (in which each item appears at most once). Furthermore, the sets of examples and patterns could then easily be represented using lists of elements of $\mathcal{P}$ or $\mathcal{E} .^{2}$

\subsection{Basic querying primitives in RDM}

The following primitives are supported by the inductive database language RDM. We provide generic definitions of the primitives that are meaningful across different pattern domains. However, we illustrate them mainly on item-sets, which results in the language RDM(IS). Throughout the paper we employ a Prolog like style and syntax. Consider the following predicates:

- +Pattern covers +Example: succeeds whenever the Pattern covers the Example.

- ?Pattern1 $<<=+$ Pattern2: succeeds whenever Pattern1 is 'more general than' Pattern2, i.e. whenever Pattern1 covers an example $e$, Pattern2 covers $e$ as well ${ }^{3}$. Also, the usual variant 'strictly more general' is $<<$.

It will be convenient to refer to the most specific pattern within the domain as bottom and to the most general one as top.

In the domain of item-sets IS (with the above sketched data types), both covers and $<<=$ correspond to the subset relation. Indeed, for item-sets $P, P 1, P 2$ and $E, P$ covers $E$ if and only if $P \subseteq E$, and $P 1<<=P 2$ if and only if $P 1 \subseteq P 2$.

Although for item-sets, covers and $<<=$ coincide this is not the case for some of the more complex domains such as DQ. Indeed, for Datalog queries, the typical 'more general than' notion corresponds to a form of $\theta$-subsumption, whereas coverage would be tested by instantiating the query with the example and answering the resulting query on the database.

${ }^{2}$ In practical implementations, it is likely that sets would be represented differently, e.g. using files.

3 The reason for employing the notation $<<=$ to denote the 'is more general than' relation is that this relation often coincides with the subset relation $\subseteq$ (or a variant thereof). The reader has to keep this interpretation in mind when reasoning about $<<=$. 
The following properties of primitives will turn out to be crucial for efficiency reasons.

Definition 5. Let $f: \mathcal{D}(\mathcal{P}) \rightarrow R$ be a function from patterns to real numbers. We say that $f$ is monotonic (resp. anti-monotonic) whenever $P<<=Q$ implies $f(P) \leq f(Q)$ (resp. $(f(P) \geq f(Q))$ for two patterns $P$ and $Q$.

Let us now extend these notions of monotonicity and anti-monotonicity to the case where $f$ is a unary predicate taking patterns as argument. The value $f(P)$ of the predicate $f$ is then 1 for those patterns $P$ for which $f(P)$ is true, and 0 for the other patterns. Under this definition the predicate $f$ defined by the clause

$f(P)$ :- P covers ex .

where ex is a specific example, is anti-monotonic.

Abusing terminology, we will sometimes talk about monotonic or anti-monotonic queries. These queries then implicitly define a unary predicate over patterns.

Sometimes it will be useful to relax the condition on coverage. For instance, one might be interested in patterns that almost cover the example. This can be realized using the following primitive.

- match(+Pattern, +Example) denotes the degree to which the Pattern matches the Example. It is required that matches $(P, e x)$ for any specific example ex is monotonic w.r.t. $<<=$.

For instance, the degree to which an item-set $P$ considered as a pattern matches an item-set $E$ considered as an example could be defined as follows.

$$
\operatorname{match}(P, E)=|P|-|P \cap E|
$$

This notion of matching might appear unnatural at first sight because it yields the value 0 when there is a perfect match and a positive integer otherwise. This notion of matching is however motivated by the monotonicity requirement, which is as we shall see, crucial for efficiency reasons.

For some applications it might also be more natural to work with a dual notion of matching, called anti-matching. The function anti-match(P,E) for itemsets could be defined as $|P \cap E|$. Anti-matching should (and in this case does) satisfy the anti-monotonicity requirement.

The typical use of the primitive match (as well of the primitives frequency, anti-match and similarity introduced below) will be in a literal of the form match(P,E) op Num where op is a comparison operator such as $<,>, \leq, \geq$, and $\mathrm{P}, \mathrm{E}$ and Num are a pattern, example and a number, respectively. Notice that for fixed E, Num and op the corresponding query behaves either monotonically or nonmonotonically.

Another desirable primitive concerns similarity.

- similarity(+Element1,+Element2): denotes the similarity between the two elements Element1 and Element2. 
Similarity among two item-sets $I$ and $J$ can be defined as

$$
\operatorname{similarity}(I, J)=\frac{2 \times|I \cap J|}{|I|+|J|}
$$

This definition has the property that the similarity between $I$ and $J$ is 1 if and only if $I$ and $J$ are identical. Similarity could be used to perform similarity based reasoning such as required by the $k$-nearest neighbor algorithm or clustering algorithm, where the basic operation is the computation of the similarity of one example to another. Unfortunately similarity is neither monotonic nor antimonotonic. This will make its efficient implementation hard.

The true data miner's favourite primitive is:

- frequency(-E, +Set,+Query): denotes the number of all elements $E$ in Set for which Query succeeds. It is required that the variable E occurs in Query. The frequency corresponds to the cardinality of the set NewSet when the predicate defineset(E,Set, Query, NewSet) (cf. below) succeeds.

Now that we have defined all the basic operations on examples and patterns, we still need to define primitives that allow us to manipulate sets of examples and of patterns.

- defineset(-E,+Set, +Query,-NewSet): succeeds when NewSet is the set of elements $E$ for which Query succeeds. It is mandatory that E occurs in Query.

For instance, the query defineset(E, DataSet, anti-match([beer,mustard,cheese], E) $\geq 2$ ), Set), succeeds if Set is the list of all examples in DataSet that have at least two items in common with [beer,mustard,cheese].

The predicate defineset could - for the domain of item-sets - be implemented using Prolog's setof0 predicate.

defineset (El, Set, Query, NewSet) :-

sefof $0(E l,($ member (El, Set), call(Query)), NewSet).

The predicate defineset is crucial to the framework as it allows us to manipulate sets of patterns and data. This predicate is RDM's way to realize the so called closure property (cf. [3]). ${ }^{4}$

\subsection{Queries, modes and safety}

It should be noted that not all queries are safe. For instance, the query ?- Pat1 $<<=$ Pat2 is unsafe because it has - in general - an infinite number of answers. To avoid this problem, queries involving patterns will typically be bounded from below (using e.g. ?- Pat $<<=$ bottom).

We address the problem in the usual Prolog manner. The above definitions of the primitives include the usual mode-declarations. Mode conform queries are safe.

${ }^{4}$ An inductive database consists of data and patterns. Furthermore there are inductive queries that can be posed to an inductive database. The closure property states that the result of an inductive query is again an inductive database. 


\section{Example queries}

In this section, we provide a number of queries to illustrate the power of the querying approach. For simplicity, the examples are illustrated over the domain IS. However, the example queries generalize to the other domains as well.

Throughout the paper we employ databases $\mathrm{d} 1$ and $\mathrm{d} 2$ which are defined as the following lists of item-sets:

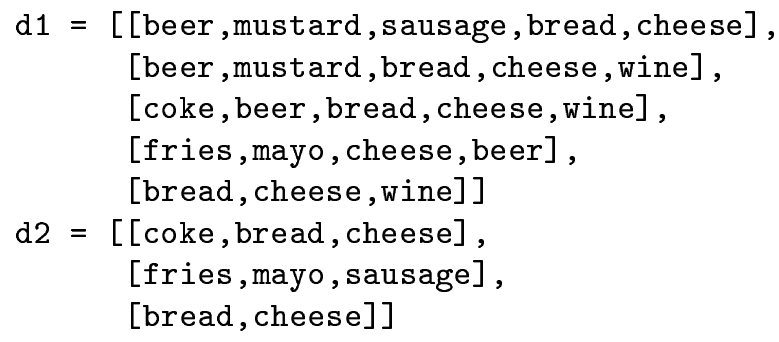

Given only these databases,

bottom $=$ [beer, bread, cheese, coke,fries, mayo, mustard, sausage, wine]

top $=[]$

\subsection{Simple queries}

Let us first specify a data mining query that returns all frequent item-sets together with their associated frequency.

(1) ?- $P \ll<$ bottom, $F$ is frequency $(E, d 1, \operatorname{covers}(P, E)), 3<F$.

The answers to this query are

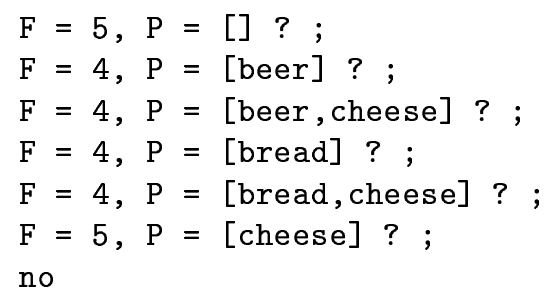

Notice that in displaying the answers, we use the traditional Prolog convention in which an answer substitution is listed explicitly and followed ended by a '?' when more answers exist. When the user inputs a ';' the next answer subsitution is listed.

A variant of query (1) succeeds only for those frequent item-sets in which beer occurs. It only returns the second and third answer.

(2) ?- $\mathrm{P} \ll<=$ bottom, [beer] $<<=\mathrm{P}, 3<\operatorname{frequency}(\mathrm{E}, \mathrm{d} 1, \operatorname{cover}(\mathrm{P}, \mathrm{E}))$. 
Another type of query generates patterns with high frequency on positives and low frequency on negatives. This is especially useful in predictive data mining.

(3) ?- $P<<=$ bottom, $3<$ frequency $(E, d 1, \operatorname{covers}(P, E))$,

frequency $(E, d 2, \operatorname{covers}(P, E))<1$.

$\mathrm{P}=$ [beer $] ?$;

$\mathrm{P}=[$ beer, cheese $] ?$;

no

The induced patterns can directly or indirectly be used for classification, i.e. to discriminate among examples from $\mathrm{d} 1$ and $\mathrm{d} 2$.

Alternatively one might impose a constraint on the accuracy directly. The following query generates patterns that are at least 80 per cent accurate. Such queries will however be harder to answer efficiently (cf. section 5.2).

(4) ?- P <<= bottom, F1 is frequency $(E, d 1, \operatorname{covers}(P, E))$,

$\mathrm{F} 2$ is frequency $(\mathrm{E}, \mathrm{d} 2, \operatorname{covers}(\mathrm{P}, \mathrm{E})), \mathrm{Acc}$ is $\mathrm{F} 1 /(\mathrm{F} 1+\mathrm{F} 2), \mathrm{Acc}>0.7$.

$\mathrm{P}=[$ beer $], \mathrm{F} 1=4, \mathrm{~F} 2=0, \mathrm{Acc}=1 ? ;$

$\mathrm{P}=[$ mustard], $\mathrm{F} 1=2, \mathrm{~F} 2=0, \mathrm{Acc}=1 ?$;

$\mathrm{P}=[$ cheese $], \mathrm{F} 1=5, \mathrm{~F} 2=2, \mathrm{Acc}=0.71 ?$;

..

Queries about similarity can be used to find those elements that are similar to a specified example.

(5) ?- E in d1, similarity (E, [beer, cheese,wine,port])> 0.5 .

$\mathrm{E}=$ [beer, mustard, bread, cheese,wine] ? ;

$\mathrm{E}=$ [coke, beer, bread, cheese, wine ] ?

$\mathrm{E}=$ [bread, cheese, wine $] ?$;

no

At this point it would also be interesting if the query language sorted its answers, e.g. according to the degree of matching or similarity. E.g. the substitutions for $\mathrm{E}$ could be sorted in such a way that the most similar examples were returned first. This is implemented in William Cohen's query language Whirl [5] which extends Datalog with a unique primitive called a 'soft join', which is related to our notion of similarity. Alternatively one might use the optimization primitives which are introduced in Section 5.1.

Let us consider also the match construct. Matching can be useful in sequence analysis in biological data (such as proteins). One can then discover all patterns that frequently match with the example to a certain extent, e.g. 
(6) ?- P $\ll=[$ bread, cheese, coke], $\operatorname{match}(\mathrm{P},[$ beer, coke $])<2$.

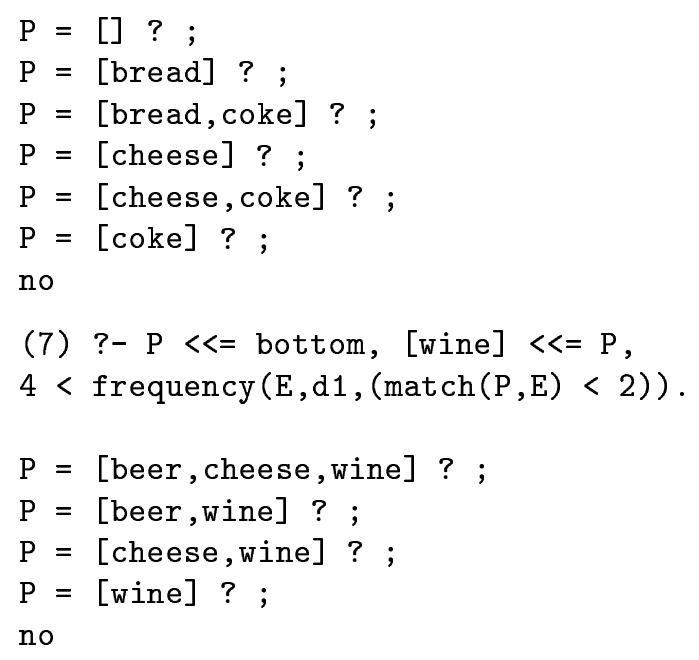

\subsection{Embedding within Prolog}

Part of the attractiveness of constraint logic programming is that constraint processing abilities are embedded within a general programming language (such as Prolog). The same is true for database languages. These can often be embedded within other programming languages as well.

In this section, we show how the above introduced primitives can be embedded within Prolog and how this allows us to easily formulate some queries of interest.

As a first illustration we show how to query for association rules over a given database (cf. [1]). It is assumed that MinAcc and MinSupport are instantiated at the time of querying to the minimum required accuracy and support, respectively.

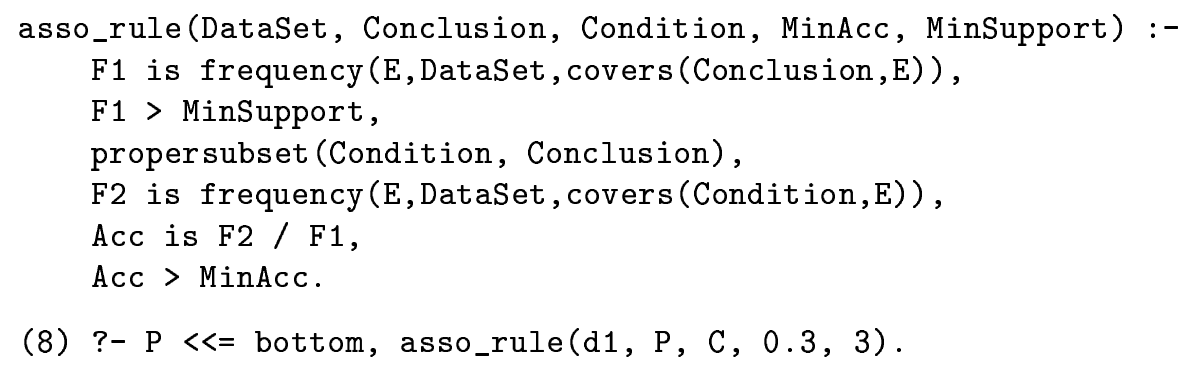

The above program can easily be extended to also include a statistical criterion for deciding whether the association rule is interesting.

Suppose that we are interested in classifying the example [beer,cheese, wine,port] as belonging to $\mathrm{d} 1$ or to $\mathrm{d} 2$. We could use the following query 
(9) ?- E1 in d1, S1 is similarity(E1, [beer, cheese,wine,port]), not(E2 in d2, S2 is similarity(E2, [beer, cheese, wine,port]), $\mathrm{S} 1<\mathrm{S} 2$ ).

This query implements the simple nearest neighbor algorithm. If the query succeeds the system predicts $d 1$, otherwise $d 2$.

Finally consider also the following complex query, which queries for two interrelated patterns.

(10) ?- P1 $\ll<=$ bottom, P2 $<<=$ bottom,

frequency $(E, d 1, P 1)>3, \operatorname{defineset}(E, d 1,(\operatorname{not} \operatorname{covers}(P 1, E)), D 2)$, frequency $(\mathrm{E}, \mathrm{D} 2, \mathrm{P} 2)>1$.

Query (10) first generates pattern P1 which has minimum frequency of 4 on the dataset $\mathrm{d} 1$. It then looks for pattern P2 which has minimum frequency of 2 on the examples not covered by pattern $\mathrm{P} 1$. Thus pattern $\mathrm{P} 2$ depends on pattern $\mathrm{P} 1$. Though such queries are in principle interesting (e.g. when learning concepts), it is - at this point - an open question as how these queries can efficiently be answered (cf. Section 4.5).

\section{Solving Queries}

So far, we have defined the semantics of the language RDM and embedded it within Prolog. It is straightforward to implement the specification of the primitives within Prolog. However, a direct implementation would not work efficiently because it would simply generate all patterns and test whether they satisfy the constraints. Below we sketch more efficient algorithms to answer queries.

The key insights that lead to efficient algorithms for answering database mining queries are 1) that the space of patterns is partially ordered by the is more general than relation, and 2) that all primitives (with the exception of similarity) are either monotonic or anti-monotonic (cf. below). From now on, we focus on such monotonic and anti-monotonic queries and will therefore ignore the similarity primitive in the rest of this section.

\subsection{Various types of queries}

Let us now investigate the introduced primitives more closely. First, the basic atoms are atoms involving the predicates covers, $<<=$ and match(P,E) op ct. Atoms of the form frequency(E,D, Query) op ct are called frequency literals.

The following basic atoms are anti-monotonic :

- Arg1 covers Arg2 : where Arg1 is a variable and Arg2 is ground

- Arg1 < = Arg2 : where Arg1 is a variable and Arg2 is ground

- match(Arg1,Arg2) op Arg3 : where Arg1 is a variable and Arg2 and Arg3 are ground and op is either $<$ or $\leq$

The basic monotonic atoms can be obtained dually: 
- Arg1 covers Arg2 : where Arg2 is a variable and Arg1 is ground

- Arg1 $<<=$ Arg2 : where Arg2 is a variable and Arg1 is ground

- match(Arg1,Arg2) op Arg3 : where Arg1 is a variable and Arg2 and Arg3 are ground and $o p$ is either $>$ or $\geq$

Furthermore, the following properties hold:

- The negation not $A$ of monotonic atom $A$ is anti-monotonic and vice versa.

- The conjunction of a set of monotonic (resp. anti-monotonic) literals querying for the same pattern variable is monotonic (resp. anti-monotonic).

- The frequency literals of the form frequency(Arg1,Arg2,Arg3) op Arg4 are anti-monotonic for a pattern variable $P$ when Arg3 is an anti-monotonic query in $P, \operatorname{Arg} 1$ is a variable occurring in $\operatorname{Arg} 3, \operatorname{Arg} 2$ and $\operatorname{Arg} 4$ are ground, and op is $>$ or $\geq$. For instance, the literal frequency $(E, d 1, \operatorname{covers}(P, E))>$ 3 is anti-monotonic for P. Frequency literals can change their status from monotonic to anti-monotonic by negating the query Arg3 or by inverting the operator op.

\subsection{The search space}

One of the most popular algorithms in data mining is the so-called level-wise search algorithm (cf. [23]). The level-wise algorithm generates all patterns $p$ that are a solution to an anti-monotonic query query $(p)$.

In order to introduce the level-wise algorithm, we need some terminology. First, let us define the notions of minimally general and maximally general elements of a set $S$ w.r.t. $<<=$.

Definition 6. Let $S$ be a set of patterns :

$-\min (S)=\{p \in S \mid \neg \exists q \in S: q<<p\}$

$-\max (S)=\{p \in S \mid \neg \exists q \in S: p<<q\}$

Second, we need operators on patterns.

Definition 7. Let $p$ be a pattern, then

- A refinement operator $\rho_{s}(p)=\max \left\{p^{\prime}\right.$ is a pattern $\left.\mid p<<p^{\prime}\right\}$.

- A generalization operator $\rho_{g}(p)=\min \left\{p^{\prime}\right.$ is a pattern $\left.\mid p^{\prime}<<p\right\}$.

For the domain IS, $\rho_{s}(p)$ contains all item-sets obtained by adding a single item to $p ; \rho_{g}(p)$ contains all item-sets obtained by deleting a single item from $p$.

The level-wise algorithm is shown below. It works iteratively and alternates between candidate evaluation (the $F$ step) and candidate generation (the $L$ ) step. In the candidate evaluation step it evaluates which of the candidates satisfy the query; the candidate generation step generates those candidates that may still satisfy the query. To this aim it employs the anti-monotonicity property together with the solutions at the previous level $F_{i}$. Notice that if $\rho_{g}(p) \nsubseteq F_{i}$ then $p$ cannot satisfy the query because of the anti-monotonicity property. 


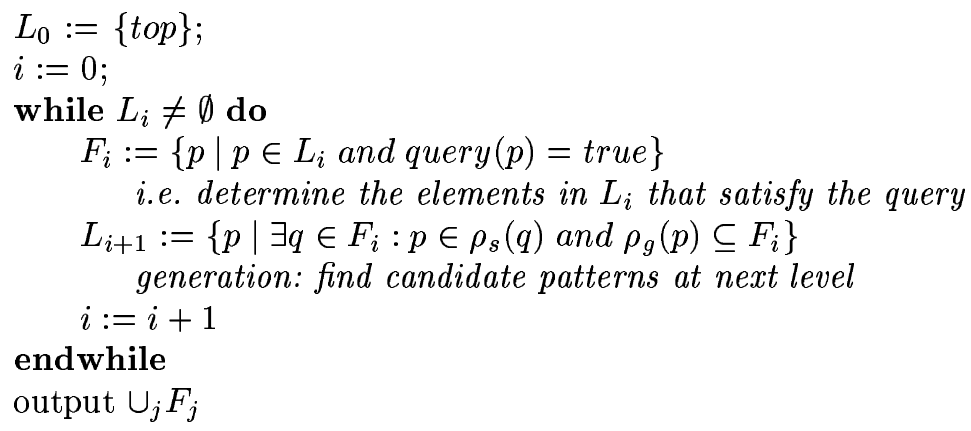

A well-known fact (cf. [23]) is that the space of solutions for anti-monotonic queries is bounded by a border $B D .{ }^{5}$

Definition 8. $B D^{+}($query $)=S(q u e r y)=\min \{p \mid p$ is a pattern and query $(p)\}$

This set corresponds to the $S$-set in Mitchell's version space framework [28]. For convenience, we use Mitchell's terminology. For an anti-monotonic query, the $S$-set completely characterizes the set of all solutions. Indeed, all patterns that are more general than a pattern in $S$ is a solution to the query as well.

Because Mitchell considers not only anti-monotonic but also monotonic constraints, Mitchell introduces also the dual of the $S$-set, which is the so called $G$-set.

Definition 9. $G(q u e r y)=\max \{p \mid p$ is a pattern and query $(p)\}$

Because of the duality, the $G$-set completely characterizes the set of solutions to a monotonic query. Furthermore, if one works with a conjunctive query that involves both monotonic and non-monotonic literals, the space of solutions is completely characterized by the two boundaries $S$ and $G$.

The space of solutions is called the version space VS(query) by Mitchell.

Definition 10. VS (query) $=\{p \mid p$ is a pattern and query $(p)\}$

The following property can be proven.

Property $1 V S($ query $)=\{p \mid p$ is a pattern and $\exists s \in S($ query $), \exists g \in$ $G($ query $): g<<=p<<=s\}$

Mitchell's original formulation of the version space considered only constraints of the form $P<<=e x$ and $\operatorname{not}(P<<=e x)$. It has been extended by various researchers, see $[27,29,16]$. For instance, Mellish extended it to also take into account constraints of the form $p<<=P$ and $\operatorname{not}(p<<=P)$. Mellish provides an algorithm that constructively computes the $S$ and $G$-sets for these 4 types of constraints. In this paper, we elaborate on the version space framework by also taking into account the frequency constraints.

\footnotetext{
${ }^{5}$ Manilla and Toivonen [23] also introduce a negative border. The negative border $B D^{-}$contains all patterns whose strict generalizations are a solution. Though it might be interesting to use the negative border in our framework, we choose not to do this for convenience.
} 


\subsection{Solving simple RDM-queries}

The version space framework is important in our context because it can be adapted to solve simple RDM queries.

Definition 11. An RDM query $?-l_{1}, \ldots, l_{n}$ is simple if all literals $l_{i} 1$ ) concern the same pattern $P$, and 2) are either monotonic or non-monotonic.

For simple queries, Property 1 holds and the space of solutions can be represented by the $S$ and $G$-sets. To illustrate this, we reformulate the answers to the above simple queries in terms of $G$ and $S$ :

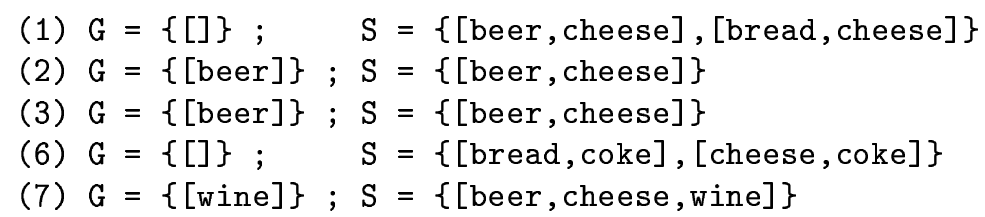

The naive way of solving a simple query would be to first split the query $q$ in two parts $q_{a}$ and $q_{m}$ corresponding to the anti-monotonic and monotonic parts respectively, and then to use the two dual versions of the level-wise algorithm. Though this approach would work it is clear that one can do better by adopting the version space algorithm.

When analyzing simple queries, the most expensive literals are those concerning frequency, because computing the frequency requires access to the data(bases). For the other literals, concerning covers, match, $<<=$, this is not necessary. Therefore, a good strategy is to first compute the $G$ and $S$ boundaries using the constraints mentioning covers, match, $<<=$ and then further shrink the version space using the frequency constraints. By doing this the hope is that the first step results in a small version space to be explored in the second step, and hence in a small number of passes through the data.

Let us first outline the algorithm for the first step. The literals for $<<=$ can be processed using Mellish's description identification algorithm. This algorithm employs the following operations patterns:

Definition 12. Let $a, b$ and $d$ be patterns :

- the greatest lower bound

$g l b(a, b)=\max \{d \mid a<<=d$ and $b<<=d\}$

- the least upper bound

$\operatorname{lub}(a, b)=\min \{d \mid d<<=a$ and $d<<=b\}$

- the most general specialisations of $a$ w.r.t. $b$

$\operatorname{mgs}(a, b)=\max \{d \mid a<<=d$ and $\operatorname{not}(d<<=b)\}$

- the most specific generalisations of $a$ w.r.t. $b$

$\operatorname{msg}(a, b)=\min \{d \mid d<<=a$ and $\operatorname{not}(b<<=d)\}$ 
function versionspace $\left(i_{1} \wedge \ldots \wedge i_{n}\right.$ : conjunctive query)

returns $S$ and $G$ defining the versionspace of $i_{1} \wedge \ldots \wedge i_{n}$

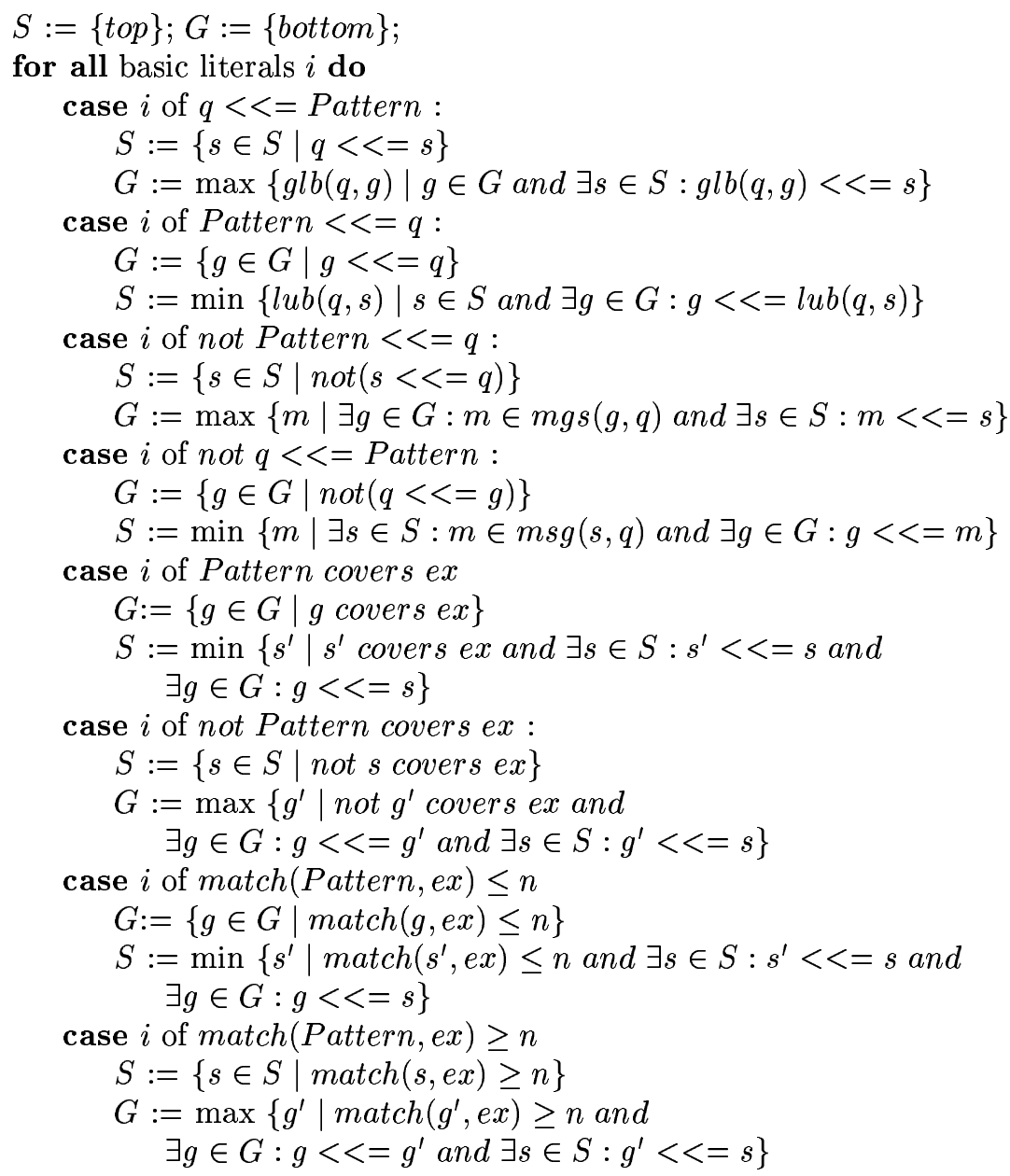

The above algorithm can be specialized according to the pattern domain under consideration. For the domain IS the specialization is rather straightforward and results in an efficient algorithm. For other domains such as DQ, the implementation of the steps for matching is more complicated. The key point about this algorithm is however that it does not require to access the data and that depending on the constraints - it results in a reduced version space.

The second step of the algorithm then deals with the frequency literals. The general outline of the algorithm is shown below. The efficient implementation of this algorithm is less straightforward. However, it turns out that we can integrate the level-wise algorithm with that of version spaces. 
for all frequency literals freq do

case $i$ is anti-monotonic :

$G:=\{g \in G \mid \operatorname{freq}(g)\}$

$S:=\min \left\{s^{\prime} \mid\right.$ freq $\left(s^{\prime}\right)$ and

$\exists s \in S: s^{\prime}<<=s$ and $\left.\exists g \in G: g<<=s^{\prime}\right\}$

case $i$ is monotonic :

$$
\begin{aligned}
& S::=\{s \in S \mid \text { freq }(s)\} \\
& G:=\max \left\{g^{\prime} \mid \text { freq }\left(g^{\prime}\right)\right. \text { and } \\
&\left.\quad \exists g \in G: g<<=g^{\prime} \text { and } \exists s \in S: g^{\prime}<<=s\right\}
\end{aligned}
$$

The first case of the second step can be implemented as follows (we assume an anti-monotonic frequency constraint freq):

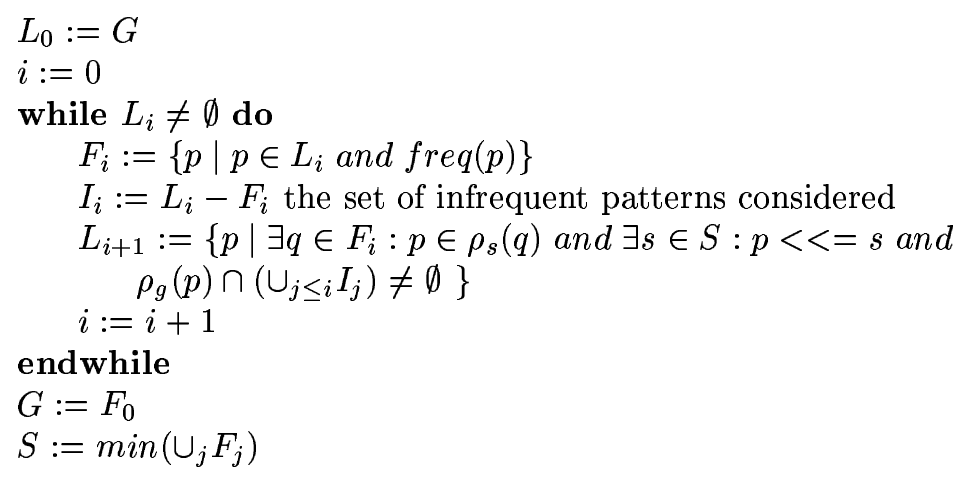

To explain the algorithm, let us first consider the case where $S=\{$ bottom $\}$ and $G=\{t o p\}$ and where we work with itemsets. In this case the refinement operator will merely add a single item to a query and the generalization operator will delete a single item from the itemset (in all possible manners). In this case, the above algorithm will behave roughly as the level-wise algorithm presented earlier. The only difference is that we keep track also of the infrequent item-sets $I_{i}$. $L_{i}$ will then contain only itemsets of size $i$. The algorithm will then repeatedly compute a set of candidate refinements $L_{i+1}$, delete those item-sets that cannot be frequent by looking at the frequency of its generalizations, and evaluate the resulting possibly frequent itemsets on the database. This process continues until $L_{i}$ becomes empty.

The basic modifications to run it in our context are concerned with the fact that we need not consider any element that is not in the already computed version space (i.e. any element not between an element of the $G$ and the $S$ set). Secondly, we have to compute the updated $S$ set, which should contain those frequent elements whose refinements are all infrequent.

Finding the updated $G$ and $S$ sets can also be realized in the dual manner. In this case, one will initialize $L_{0}$ with the elements of $S$ and proceed otherwise completely dual. The resulting algorithm is shown below. 
Whether the top down or bottom up version will work more efficiently is likely to depend on the application and query under consideration. At this point it remains an open question as to when which strategy will work more efficiently.

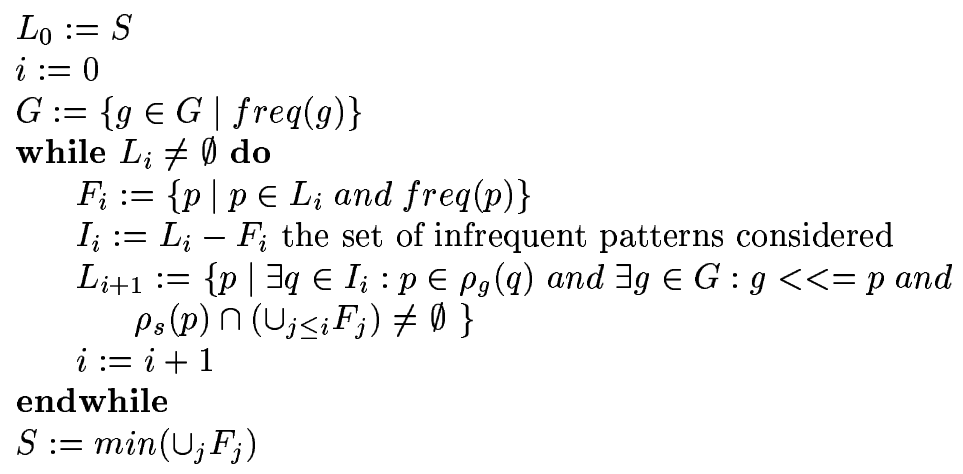

Finally, it is also easy to modify the above algorithms (exploiting the dualities) in order to handle monotonic frequency atoms (i.e. the second case in the algorithm for the second step).

Whereas in this section we have adopted the standard level-wise algorithm to search for the borders, it would also be possible to adopt more efficient algorithms such as e.g. the randomized ones proposed in [22].

\subsection{The proof of the concept}

In $[10,20,21]$ we present an implementation, called MolFea, of the level-wise version space algorithm for use in molecular applications. The examples in MolFea correspond to the $2 \mathrm{D}$ structure of chemical compounds, i.e. they are essentially graphs. Patterns in MolFea are molecular fragments, i.e. linearly connected sequences of atoms and bonds.

Furthermore, we have performed various experiments with MolFea on challenging data sets. In one of the experiments [21], we have discovered fragments of interest in a database containing information on the HIV activity of over 40000 chemicals. MolFea did find long patterns (of about 25 atom/bonds) in relatively short time (a couple of hours on Linux workstation - Pentium III). As compared to state-of-the-art inductive logic programming systems (such as e.g. Dehaspe's Warmr [7]), which have also been applied to this type of problem, MolFea compares favourable in that it handles much more data in much less time and also discovers longer patterns. Indeed, typical experiments reported in the literature indicate that inductive logic programming systems handle a few hundred compounds, find patterns of length 7 or 8 , and require several days of cpu-time.

This provides evidence that the level-wise version space algorithm is an effective solver for database mining. 


\subsection{Optimising the algorithms}

Various optimisations to the algorithm seem possible and worthy of further investigation.

Instead of processing the frequency literals independent of each other, one might rather combine these so that only one pass through the search space is necessary. Indeed, consider the following query :

(11) $?-P<<=$ bottom, frequency $(E, d 1, \operatorname{covers}(P, E))>3$, frequency $(\mathrm{E}, \mathrm{d} 1,(\operatorname{mat} c h(\mathrm{P}, \mathrm{E})<2))>4$.

This query could be answered by taking as constraint the conjuction of the two frequency literals and then performing one pass through the search space. Executing the query in this way may be more efficient.

This example illustrates that reasoning about queries and their execution will be beneficial. To this aim, one could employ notions such as query simplification, equivalence, implication, redundancy, etc. All of these standard notions could essentially be applied to the framework of RDM. Some of these notions have already been worked out for related languages such as MINESQL (cf. e.g. [26, 13]). For RDM, this remains however a topic for further research.

\subsection{Solving more complex RDM queries}

So far we have only dealt with simple RDM queries. We have not considered the similarity literals, because they are neither monotonic nor anti-monotonic. Therefore, it seems natural (though inefficient) to compute these using a generate-andtest strategy after the $G$ and $S$ borders have been computed for the rest of the query.

What also remains an open question is how to process queries such as the one in query (10), which involves two patterns. The problem is how to capture the dependencies among the two patterns within the version space model. Extensions to the basic version space model are necessary to realize this. These are also a topic for further research.

\section{Extensions to the basic engine and language}

\subsection{Optimization primitives}

Two primitives that seem especially useful are min and max. Indeed, one could imagine being interested in those patterns that satisfy a number of constraints and in addition have maximum frequency on a certain dataset or are minimally general. Let us therefore define:

$-\max (p(P), \operatorname{term}(P))$ (resp. min) takes as argument a query $p(P)$ where $P$ is a pattern variable and a term term $(P)$ to be optimized. The predicate $p$ imposes constraints on the pattern $P$ and term $(P)$ specifies the criterion that should be maximized (resp. minimized). term $(P)$ should be a monotonic or 
anti-monotonic term or literal in P. The optimization literal then succeeds for those patterns satisfying the constraints imposed by $p$ and being optimal w.r.t. $\operatorname{term}(P)$.

As an example consider the following query :

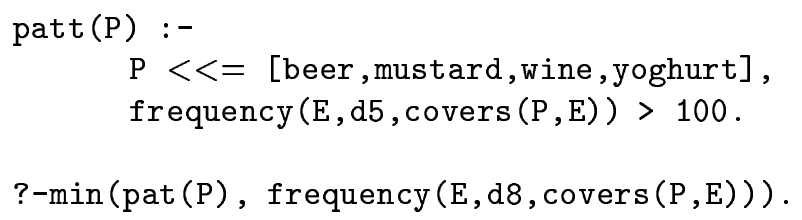

The predicate patt succeeds for all patterns involving beer, mustard, wine and yoghurt with a frequency on d5 that is larger than 100 . The optimisation literal then selects among the patterns that satisfy patt, those with minimal frequency in d8. , Within the sketched solver for simple queries, it is relatively easy to accommodate this type of optimization constructs. Indeed, because the optimization terms should be monotonic or anti-monotonic, one only needs to consider the maximum (resp. minimum) elements in the solutions to p. So, to answer a query containing an optimization literal, one first computes the $S$ and $G$ sets for p. If the criterion to be optimized is generality, then the solutions are given directly by either $S$ or $G$ (depending on whether one wishes to minimize or to maximize). If on the other hand one wishes to optimize w.r.t. frequency then one needs to compute the frequency of all elements in either $G$ or $S$. The answers to the queries are then those patterns within either $G$ or $S$ that are optimal.

\section{$5.2 \quad$ Heuristic solvers}

So far, we have described complete solvers, which all solutions within the specified constraints. However, completeness often comes at a (computational) cost. Therefore, complete solvers may not always be desirable. There are at least two situations already encountered where this might be the case.

First, the provided primitives for optimisation were so far quite simple. Also, the criterion one may want to optimize is not necessarily frequency. Indeed, one is often more interested in accuracy, or entropy, etc. To optimize w.r.t. accuracy (as in query (4)) one cannot employ the above sketched method because accuracy involves combining maximum frequency on positives and minimum frequency on negatives. Hence, optimal patterns might lie in the middle of the version space.

Second, there is a discrepancy between answering simple queries and answering queries over multiple inter-related patterns (as in query (11)). There is good hope and evidence that the former solver is reasonably efficient, but it is also clear that the latter one is much less efficient, because it merely enumerates all the possibilities.

Therefore, we need to extend the current solver with heuristic methods. This situation is again akin to what happens in constraint logic programming (cf. [25]). 
The effect of heuristic methods would be that queries get a heuristic answer, that some solutions are not found, and that suboptimal solutions are generated. Of course, in such cases the user should be aware of this. When allowing for heuristic methods, it becomes possible to extend the database mining engine with various well-known database mining algorithms, such as a beam-search procedure to greedily find the most interesting clauses in predictive modelling. In this context, any-time algorithms would also be quite effective.

\subsection{The knowledge discovery cycle}

Knowledge discovery in databases typically proceeds in an iterative manner. Data are selected, possibly cleaned, formatted, input in a data mining engine, and results are analysed and interpreted. As first results often can be improved, one would typically re-iterate this process until some kind of a local optimum has been reached (cf. [12]).

Because knowledge discovery is an iterative process data mining tools should support this process. One consequence of the iterative nature of knowledge discovery in our context is that many of the queries formulated to the database mining engine will be related. Indeed, one can imagine that various queries are similar except possibly for some parameters such as thresholds, data sets, pattern syntax, etc. The relationships among consecutive queries posed to the data mining engine should provide ample opportunities for optimization. The situation is - to some extent - akin to the way that views are dealt with in databases (cf. [11]). Views in databases are similar to patterns in data mining in that both constructs are virtual data structures, i.e. they do not physically exist in the database. Both forms of data can be queries and it is the task of the engines to efficiently answer questions concerning these virtual constructs.

Answering queries involving views can be realized essentially in two different ways. First, one can materialize views, which means that one generates the tuples in the view relation explicitly, and then processes queries as if a normal relation were queried. Second, one can perform query modification, which means that any query to a view is 'unfolded' into a query over the base relations. The advantage of materialization is that new queries are answered much faster whereas the disadvantage is that one needs to recompute or update the view whenever something changes in the underlying base relations. At a more general level, this corresponds to the typical computation versus storage trade-off in computer science.

These two techniques also apply to querying patterns in data mining. Indeed, if consecutive queries are inter-related, it would be useful to store the results (and possibly also the intermediate results) of one query in order to speed up the computation of the latter ones. This corresponds to materializing the patterns (together with accompanying information). Doing this would result in effective but fairly complicated solvers. 


\section{Related Work}

$\mathrm{RDM}$ is related to other proposals for database mining query languages such as e.g. $[26,17,14,13,3]$. However, it differs from these proposals in a number of aspects. First, due to the use of deductive databases as the underlying database model, RDM allows - in principle - to perform pattern discovery over various domains, such as e.g. item-sets, sequences, graphs, datalog queries, ... Secondly, a number of new and useful primitives are foreseen. Using RDM one is not restricted to finding frequent patterns, but one may also look for infrequent ones with regards to certain sets of (negative) examples. One can also require that certain examples are (resp. are not) covered by the patterns to be induced. Thirdly and most importantly, RDM allows to combine different primitives when searching for patterns. Finally, its embedding within Prolog puts database mining on the same methodological grounds as constraint programming.

As another contribution, we have outlined an efficient algorithm for answering complex database mining queries. This algorithm integrates the principles of the level-wise algorithm with those of version spaces and thus provides evidence that RDM can be executed efficiently. It also provides a generalized theoretical framework for data mining. The resulting framework extends the borders in the level-wise techniques sketched by [23], who link the level-wise algorithm to the $\mathrm{S}$ set of Mitchell's version space approach but do not further exploit the version space model as we do here. An implementation of the level-wise versionspace algorithm for use in molecular applications has been implemented [20,21] and the results obtained are promising.

Finally, the author hopes that this work provides a new perspective for data mining, which is grounded in the methodology of computational logic. The hope is that this will result in a clear separation of the declarative from the procedural aspects in data mining.

\section{Acknowledgements}

This work was partially supported by the EU IST project cInQ. The author is grateful to Stefan Kramer, Jean-Francois Boulicaut and the anonymous reviewers for comments, suggestions and discussions on this work.

\section{References}

1. R. Agrawal, T. Imielinski, A. Swami. Mining association rules between sets of items in large databases. In Proceedings of ACM SIGMOD Conference on Management of Data, pp. 207-216, 1993.

2. E. Baralis, G. Psaila. Incremental Refinement of Mining Queries. In Mukesh K. Mohania, A. Min Tjoa (Eds.) Data Warehousing and Knowledge Discovery, First International Conference DaWaK '99 Proceedings. Lecture Notes in Computer Science, Vol. 1676, Springer Verlag, pp. 173-182, 1999. 
3. Jean-Francois Boulicaut, Mika Klemettinen, Heikki Mannila: Querying Inductive Databases: A Case Study on the MINE RULE Operator. In Proceedings of PKDD98, Lecture Notes in Computer Science, Vol. 1510, Springer Verlag, pp. 194-202, 1998.

4. I. Bratko. Prolog Programming for Artificial Intelligence. Addison-Wesley, 1990. 2nd Edition.

5. W. Cohen, Whirl : a word-based information representation language. Artificial Intelligence, Vol. 118 (1-2), pp. 163-196, 2000.

6. L. Dehaspe, H. Toivonen and R.D. King. Finding frequent substructures in chemical compounds, in Proceedings of KDD-98, AAAI Press, pp. 30-36, 1998.

7. L. Dehaspe, H. Toivonen. Discovery of Frequent Datalog Patterns, in Data Mining and Knowledge Discovery Journal, Vol. 3 (1), pp. 7-36, 1999.

8. L. De Raedt, An inductive logic programming query language for database mining (Extended Abstract), in Proceedings of Artificial Intelligence and Symbolic Computation, Lecture Notes in Artificial Intelligence, Vol. 1476, Springer Verlag, pp. 1-13, 1998.

9. L. De Raedt, A Logical Database Mining Query Language. in Proceedings of the 10th Inductive Logic Programming Conference, Lecture Notes in Artificial Intelligence, Vol. 1866, Springer Verlag, pp. 78-92, 2000.

10. L. De Raedt, S. Kramer, The level-wise version space algorithm and its application to molecular fragment finding, in Proceedings of the Seventeenth International Joint Conference on Artificial Intelligence, Morgan Kaufmann, pp. 853-862, 2001.

11. Elmasri, R. and Navathe, S. Fundamentals of database systems. Benjamin Cummings. 1994.

12. Usama M. Fayyad, Gregory Piatetsky-Shapiro, Padhraic Smyth, Ramasamy Uthurusamy (Eds.). Advances in Knowledge Discovery, The MIT Press, 1996.

13. F. Giannotti, G. Manco: Querying Inductive Databases via Logic-Based UserDefined Aggregates. In Proceedings of PKDD 99, Lecture Notes in Artificial Intelligence, Vol. 1704, Springer Verlag, pp. 125-135, 1999.

14. J. Han, Y. Fu, K. Koperski, W. Wang, and O. Zaiane, DMQL: A Data Mining Query Language for Relational Databases, in SIGMOD'96 Workshop on Research Issues on Data Mining and Knowledge Discovery, Montreal, Canada, June 1996.

15. J. Han, L. V. S. Lakshmanan, and R. T. Ng, Constraint-Based, Multidimensional Data Mining, Computer, Vol. 32(8), pp. 46-50, 1999.

16. H. Hirsh. Generalizing Version Spaces. Machine Learning, Vol. 17(1), pp. 5-46 (1994).

17. T. Imielinski and H. Mannila. A database perspectivce on knowledge discovery. Communications of the ACM, Vol. 39(11), pp. 58-64, 1996.

18. T. Imielinski, A. Virmani, and A. Abdulghani. Application programming interface and query language for database mining. In Proceedings of KDD 96. AAAI Press, pp. 256-262, 1996.

19. Robert A. Kowalski. Algorithm = Logic + Control. Communications of the ACM, 22(7), pp. 424-436, 1979.

20. Kramer, S. and De Raedt, L. Feature Construction with Version Spaces for Biochemical Applications, in Proceedings of the Eighteenth International Conference on Machine Learning, Morgan Kaufmann, 2001.

21. Kramer, S., De Raedt, L., Helma, C. Molecular Feature Mining in HIV Data, in Proceedings of the Seventh ACM SIGKDD International Conference on Knowledge Discovery and Data Mining, ACM Press, pp. 136-143, 2001. 
22. D. Gunopulos, H. Mannila, S. Saluja: Discovering All Most Specific Sentences by Randomized Algorithms. In Foto N. Afrati, Phokion Kolaitis (Eds.): Database Theory - ICDT '97, 6th International Conference, Lecture Notes in Computer Science, Vol. 1186, Springer Verlag, pp. 41-55, 1997.

23. H. Mannila and H. Toivonen, Levelwise search and borders of theories in knowledge discovery, Data Mining and Knowledge Discovery, Vol. 1(3), pp. 241-258, 1997.

24. H. Mannila. Inductive databases. in Proceedings of the International Logic Programming Symposium, The MIT Press, pp. 21-30, 1997.

25. Marriott, K. and Stuckey, P. J. Programming with constraints : an introduction. The MIT Press. 1998.

26. R. Meo, G. Psaila and S. Ceri, An extension to SQL for mining association rules. Data Mining and Knowledge Discovery, Vol. 2 (2), pp. 195-224, 1998.

27. C. Mellish. The description identification algorithm. Artificial Intelligence, Vol. 52 (2), pp, 151-168, 1990.

28. T. Mitchell. Generalization as Search, Artificial Intelligence, Vol. 18 (2), pp. 203$226,1980$.

29. G. Sablon, L. De Raedt, and Maurice Bruynooghe. Iterative Versionspaces. Artificial Intelligence, Vol. 69(1-2), pp. 393-409, 1994.

30. A. Inokuchi, T. Washio, H. Motoda. An Apriori-based algorithm for mining frequent substructures from graph data. in D. Zighed, J. Komorowski, and J. Zyktow (Eds.) Proceedings of PKDD 2000, Lecture Notes in Artificial Intelligence, Vol. 1910, Springer-Verlag, pp. 13-23, 2000.

This article was processed using the ${ }^{A} \mathrm{~T}_{\mathrm{E}} \mathrm{X}$ macro package with LLNCS style 